\title{
MODEL-BASED IMAGE RECONSTRUCTION FOR DUAL-ENERGY X-RAY CT WITH FAST KVP SWITCHING
}

\author{
Wonseok Huh and Jeffrey A. Fessler \\ Department of Electrical Engineering and Computer Science \\ The University of Michigan \\ 1301 Beal Avenue, Ann Arbor, MI 48109-2122
}

\begin{abstract}
The most recent generation of X-ray CT systems can collect dual energy (DE) sinograms by rapidly switching the $\mathrm{X}$-ray tube voltage between two levels for alternate projection views. This reduces motion artifacts in DE imaging, but yields sinograms that may be angularly under-sampled. This paper describes an iterative algorithm for statistical image reconstruction of material component images (e.g., soft tissue and bone) directly from such under-sampled DE data, without resorting to the interpolation operations required by conventional DE reconstruction methods.

Keywords: model-based image reconstruction, dualenergy X-ray computed tomography, penalized likelihood.
\end{abstract}

\section{INTRODUCTION}

In the field of X-ray CT, there is increasing interest in enhancing the information provided in the images through dualenergy imaging. Dual energy (DE) CT imaging was first proposed over 30 years ago [1], but only recently became available for routine use in clinical CT systems. Various technological advances have brought renewed interest in DE CT, such as CT systems with two X-ray sources and photon counting detectors with energy selectivity. Very recently, commercial systems with fast $\mathrm{kVp}$ switching have become available, extending an idea that previously existed only in prototype systems [2]. This paper describes a model-based approach to DE image reconstruction for such systems.

The conventional approach to DE CT imaging is the "dual rotate" mode where the source is rotated around the patient at one source voltage setting to collect a full sinogram, and then the source voltage is changed (as quickly as the hardware permits) and the source is rotated around again with the new $\mathrm{kVp}$ to collect a second full sinogram. Using these two full sinograms, one can reconstruct separate images of two material components (such as soft tissue and bone) using sinogram material decomposition followed by FBP image reconstruction [1]. Model-based image reconstruction methods for fully

This work was supported in part by NIH/NCI grant 1R01CA115870. sampled sinograms have also been proposed under monoenergetic $[3,4]$ and polyenergetic models [5,6]. A drawback of this conventional "dual rotate" mode of DE CT is that the object may move between the two acquisitions, leading to inconsistencies between the two sinograms that can manifest as severe artifacts in the reconstructed images.

To reduce motion effects, the "fast $\mathrm{kVp}$ switching" mode alternates between high and low X-ray source tube voltages for the projection views. This allows DE data to be collected in a single rotation, so the motion artifacts should be comparable to those of conventional CT imaging. Modern CT systems rotate in less than 0.5 seconds, and collect about 1000 projection views, so the $\mathrm{X}$-ray tube high voltage must be switched at $\mathrm{kHz}$ rates repeatably, which is a challenge that has been met only recently. In this switching mode, two sinograms are collected with only half as many projection views as could be collected otherwise. Conventional sinogram domain DE decomposition methods require each ray to be measured twice by two different spectra (two different tube voltages), but in the fast switching mode, the even projection views are at one voltage and the odd views are at the other voltage.

The typical solution to this problem in fast switching DE imaging is to interpolate both sinograms in the angular direction to fill in the "missing" views. Then one can apply conventional DE decomposition followed by FBP reconstruction. However, such interpolation might compromise spatial resolution. Furthermore, DE decomposition is a noise-amplifying process, so statistical image reconstruction methods have the potential to improve image quality significantly relative to FBP in DE imaging [5]. This paper proposes a model-based image reconstruction method for DE CT that reconstructs the two material component images (e.g., soft tissue and bone) directly from the under-sampled sinograms without any interpolation. We report preliminary simulation results suggesting that this iterative method has the potential to improve image quality compared to the conventional interpolate/FBP approach. 


\section{DUAL-ENERGY RECONSTRUCTION}

\subsection{Models}

\subsubsection{Measurement model}

Let $y_{m i}$ denote the CT measurement for the $i$ th ray for the $m$ th incident spectrum, $m=1, \ldots, M_{0}, i=1, \ldots, N_{\mathrm{d}}$. For $\mathrm{DE} \mathrm{CT}, M_{0}=2$. The measurement means are related to the line-integral projections of the object's attenuation via Beer's law. We assume that the measurements are random variables with the following ensemble means:

$$
\bar{y}_{m i} \triangleq \int I_{m i}(\mathcal{E}) \exp \left(-\int_{\mathcal{L}_{i}} \mu(\vec{x}, \mathcal{E}) \mathrm{d} \ell\right) \mathrm{d} \mathcal{E}+r_{m i}
$$

where $\int_{\mathcal{L}_{i}} \cdot \mathrm{d} \ell$ denotes the line integral along the $i$ th ray, and $\mu(\vec{x}, \mathcal{E})$ denotes the linear attenuation coefficient (LAC) of the object being scanned at the spatial location $\vec{x}, I_{m i}(\mathcal{E})$ denotes the product of the $m$ th incident source spectrum and the detector gain for the $i$ th ray, and $r_{m i}$ denotes additive background contributions such as room background, dark current, and scatter. We treat $I_{m i}(\mathcal{E})$ and $r_{m i}$ as known nonnegative quantities [7-9].

\subsubsection{Object model}

The measurements are finite whereas $\mu$ is a continuous function of spatial location $\vec{x}$ and energy, $\mathcal{E}$. Thus for reconstruction we parameterize the LAC using basis functions that are separable in the spatial and energy dimension as follows [1]:

$$
\mu(\vec{x}, \mathcal{E})=\sum_{l=1}^{L_{0}} \sum_{j=1}^{N_{\mathrm{p}}} \beta_{l}(\mathcal{E}) b_{j}(\vec{x}) \rho_{l j}
$$

where $\beta_{l}(\mathcal{E})$ denotes the energy-dependent mass-attenuation coefficient (MAC) of the $l$ th material type (units $\mathrm{cm}^{2} / \mathrm{g}$ ), $\left\{b_{j}(\vec{x})\right\}$ are (unitless) spatial basis functions such as square pixels, and $\rho_{l}=\left(\rho_{l 1}, \ldots, \rho_{l N_{\mathrm{p}}}\right)$ denotes the vector of unknown density values of $l$ th material type (units $\mathrm{g} / \mathrm{cm}^{3}$ ) for each of the $N_{\mathrm{p}}$ voxels. In DE CT, we usually choose $L_{0}=2$ e.g., soft tissue (water) and bone. We use tabulated MAC values for water and bone [10].

Most conventional approaches to DE CT imaging have estimated the object, $\left\{\rho_{l}\right\}_{l=1}^{L_{o}}$, from fully sampled measurements, $\left\{y_{m i}\right\}_{i=1}^{N_{\mathrm{d}}}$. In this paper, the goal is to reconstruct the object from under-sampled sinograms that are collected by alternating X-ray source voltage over a single rotation. Therefore, the proposed method estimates $\left\{\rho_{l}\right\}_{l=1}^{L_{0}}$ from $\left\{y_{m i}\right\}_{i \in \mathcal{I}_{m}}$ where the sets of indexes $\left\{\mathcal{I}_{m}\right\}_{m=1}^{M_{0}}$ are a partition of whole index set $I=\left\{1, \ldots, N_{\mathrm{d}}\right\}$. In particular, for DE CT with fast $\mathrm{kVp}$ switching between source voltages, $\mathcal{I}_{1}$ and $\mathcal{I}_{2}$ correspond to the rays in the odd and even projection views respectively. Using (1), (2), we rewrite the ensemble means of the measurements as follows:

$$
\begin{aligned}
\bar{y}_{m i}(\rho) & =I_{m i} \mathrm{e}^{-f_{m i}(\rho)}+r_{m i} \\
f_{m i}(\rho) & \triangleq-\log v_{m i}(\rho) \\
v_{m i}(\rho) & \triangleq \int p_{m i}(\mathcal{E}) \mathrm{e}^{-\beta(\mathcal{E}) \cdot \mathbf{s}_{i}(\rho)} \mathrm{d} \mathcal{E},
\end{aligned}
$$

for $m=1, \ldots, M_{0}, l=1, \ldots, L_{0}$, and $i \in \mathcal{I}_{m}$, where $I_{m i}=$ $\int I_{m i}(\mathcal{E}) \mathrm{d} \mathcal{E}$ denotes the total intensity for the $m$ th incident spectrum and the $i$ th ray, and we define the sinogram vector $\mathbf{s}_{i}$ as follows:

$$
\begin{array}{lll}
p_{m i}(\mathcal{E}) \triangleq I_{m i}(\mathcal{E}) / I_{m i}, & \beta(\mathcal{E}) \triangleq\left(\beta_{1}(\mathcal{E}), \ldots, \beta_{L_{0}}(\mathcal{E})\right), \\
s_{i l}(\rho) \triangleq\left[A \rho_{l}\right]_{i}, & \mathbf{s}_{i}(\rho) \triangleq\left(\mathbf{s}_{i 1}(\rho), \ldots, \mathbf{s}_{i L_{0}}(\rho)\right),
\end{array}
$$

where $A$ denotes the $N_{\mathrm{d}} \times N_{\mathrm{p}}$ system matrix having elements

$$
a_{i j} \triangleq \int_{\mathcal{L}_{i}} b_{j}(\vec{x}) \mathrm{d} \ell .
$$

\subsection{Conventional Interpolation/FBP approach}

The usual estimate of the $f_{m i}$ values is to invert (4):

$$
\hat{f}_{m i} \triangleq-\log \left(\operatorname{smooth}\left\{\frac{Y_{m i}-r_{m i}}{I_{m i}}\right\}\right) \text {, for } i \in \mathcal{I}_{m} \text {, }
$$

where radial smoothing is often included to reduce noise, e.g. [11]. Using angular interpolation, one can reconstruct $\hat{f}_{m i}$ for all $i=1,2, \ldots, N_{\mathrm{d}}$. For the results shown below, we simply averaged the two nearest projection views for interpolation. Then one applies conventional DE decomposition [1], followed by FBP reconstruction. This approach is fast but suboptimal.

\subsection{Penalized Weighted Least Square (PWLS) approach}

Instead of estimating $\rho$ by using interpolation, we propose to estimate $\rho$ directly by including a spatial roughness penalty $\mathrm{R}(\rho)$ in the penalized-likelihood cost function:

$$
\begin{gathered}
\hat{\rho}=\underset{\rho \geq 0}{\arg \min } \Psi(\rho) \\
\Psi(\rho) \triangleq \sum_{m=1}^{M_{0}} \sum_{i \in \mathcal{I}_{m}} \frac{w_{m i}}{2}\left(\hat{f}_{m i}-f_{m i}(\rho)\right)^{2}+R(\rho),
\end{gathered}
$$

where $w_{m i}$ denotes weights that we define as follows:

$$
w_{m i}=Y_{m i}, \text { for } i \in \mathcal{I}_{m} \text {. }
$$

These weights are a reasonable choice because CT measurements are approximately Poisson distributed and in the absence of smoothing the approximate variance of $\hat{f}_{m i}$ is

$$
\operatorname{Var}\left(\hat{f}_{m i}\right) \approx \frac{\operatorname{Var}\left(Y_{m i}\right)}{\left(\bar{y}_{m i}-r_{m i}\right)^{2}} .
$$


Note that (8) uses only the measured rays $\left(i \in \mathcal{I}_{m}\right)$; no interpolation is used. The regularizing penalty term in (8) is given by the following:

$$
R(\rho)=\sum_{l=1}^{L_{0}} \sum_{j=1}^{N_{\mathrm{p}}} \sum_{k \in \mathcal{N}_{j}} \psi\left(\rho_{l j}-\rho_{l k}\right),
$$

where $\psi$ is a potential function and $\mathcal{N}_{j}$ is a neighborhood of pixel $j$. For $\psi$ we used a hyperbola [12] and the modified regularizer in [13] to provide uniform spatial resolution. Here we minimized the cost function in (8) using 100 iterations of a conjugate graduate method with a monotone line search technique [14]. This could be accelerated greatly by using ordered subsets [15]. We initialized the iterations using the object estimated by the interpolation/FBP method in Section 2.2 .

\section{RESULTS}

To evaluate the feasibility of the proposed methods for image reconstruction, we performed a computer simulation of dualenergy CT scans. The reconstructed images were $512 \times 512$ with $0.1 \times 0.1 \mathrm{~cm}^{2}$ pixel size and the projection space was 888 radial samples $\times 984$ angular views. We applied the conventional dual-energy interpolation/FBP reconstruction method, and the proposed regularized PWLS method with source voltages $80 \mathrm{kVp}$ and $140 \mathrm{kVp}$. We investigated 10 different numbers of incident photons per ray from $1 \times 10^{5}$ to $1 \times 10^{6}$.

Fig. 1 shows the density maps of the components: soft tissues and bone mineral and the estimated object of the two methods with $I_{0}=I_{m i}=10^{5}, i \in \mathcal{I}_{m}$. The PWLS images have reduced streak artifacts and lower noise than the conventional FBP images.

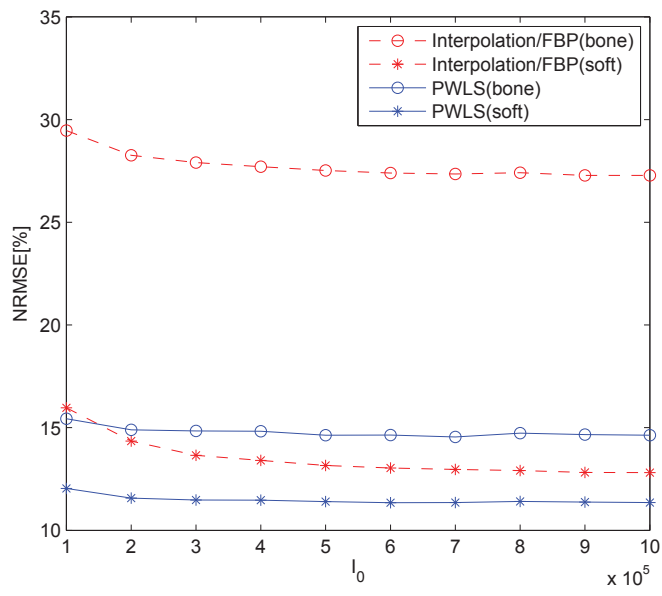

Fig. 2. NRMSE of estimated density maps: Interpolation/FBP method, and proposed PWLS algorithm.
Fig. 2 shows that the proposed PWLS method reduces significantly the NRMSE of the soft tissue and bone images compared to the conventional interpolation/FBP method.

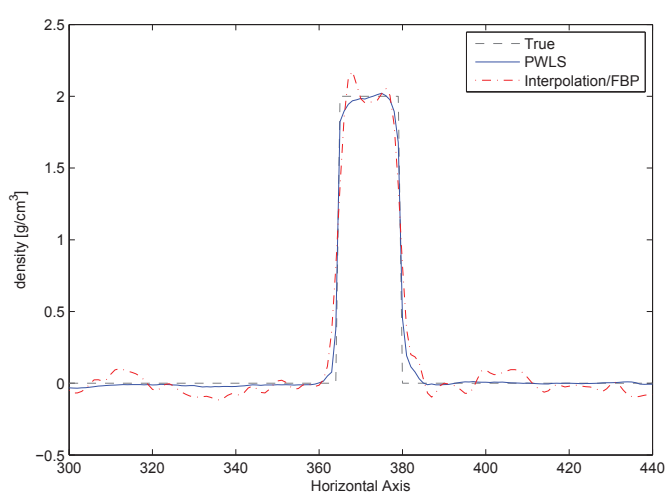

Fig. 3. Horizontal profile through the estimated bone images of the two methods and the true bone image.

Fig. 3 illustrates that the proposed PWLS method has lower noise than FBP without compromising the spatial resolution near object edges.

For completeness, we also applied the PWLS method to the interpolated sinogram data. Fig. 1(d) and (h) show their resulting component images. The global NRMSE of the soft tissue and bone components were $14.8 \%$ and $11.9 \%$ respectively, compared to $14.6 \%$ and $11.9 \%$ for PWLS applied to the sinograms without interpolation. When implemented efficiently, applying PWLS to interpolated data requires approximately twice the compute time per iteration as PWLS for the original under-sampled data.

\section{CONCLUSION}

We presented an iterative regularized PWLS algorithm for DE CT reconstruction from the type of under-sampled DE data that is collected by fast $\mathrm{kVp}$ switching $\mathrm{CT}$ systems. Unlike other DE CT algorithms, the proposed method estimates material component images directly from only half as many projection views without any interpolation operation. The experiments show that the proposed method yields images with lower NRMS error than the conventional interpolation/FBP approach in Fig. 2. Our next step is to evaluate the method with real data and to investigate penalized-likelihood methods and to analyze the spatial resolution and noise properties.

\section{ACKNOWLEDGE}

The authors gratefully acknowledge Paul Kinahan and Adam Alessio for discussions regarding DE CT. 


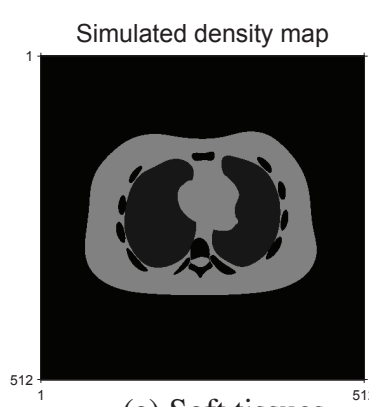

(a) Soft tissues

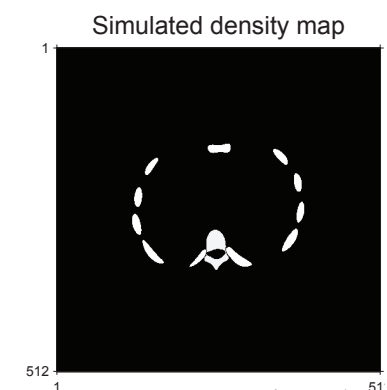

(e) Bone minerals

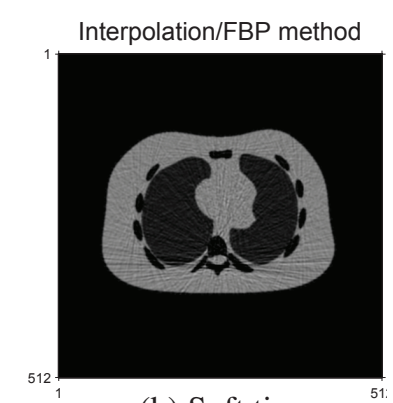

(b) Soft tissues

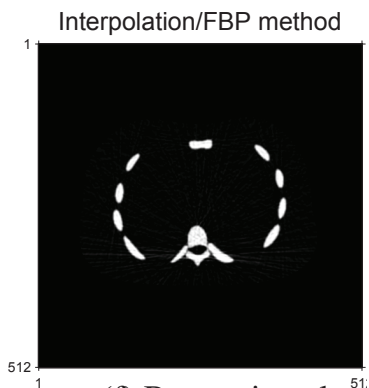

(f) Bone minerals

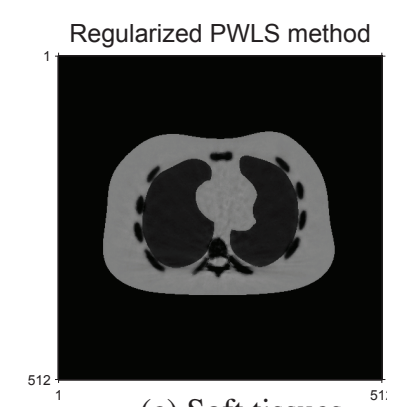

(c) Soft tissues

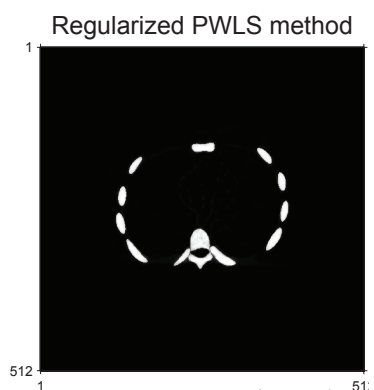

(g) Bone minerals ${ }^{5}$

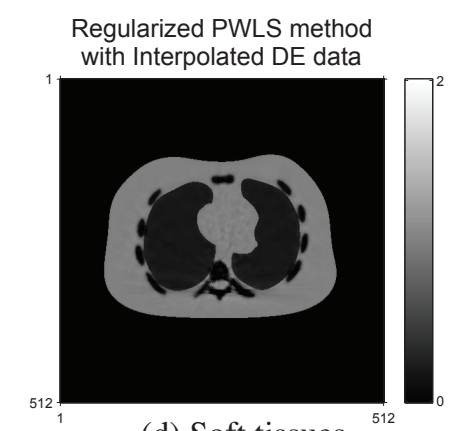

(d) Soft tissues

Regularized PWLS method with Interpolated DE data

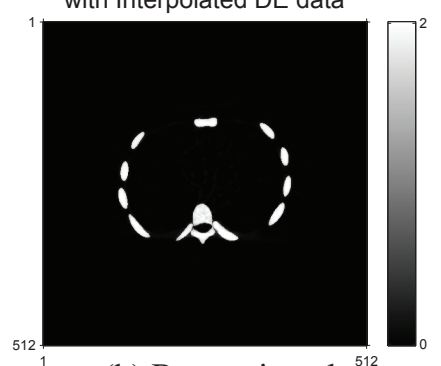

(h) Bone minerals

Fig. 1. First column: Two component simulated densities. Second column: Interpolation/FBP method with $I_{0}=10^{5}$. Third column: Regularized PWLS method with $I_{0}=10^{5}$. Fourth column: Regularized PWLS method with interpolated DE data.

\section{REFERENCES}

[1] R. E. Alvarez and A. Macovski, "Energy-selective reconstructions in X-ray computed tomography," Phys. Med. Biol., vol. 21, no. 5, pp. 733-44, Sept. 1976.

[2] W. A. Kalender, W. H. perman, J. R. Vetter, and E. Klotz, "Evaluation of a prototype dual-energy computed tomographic apparatus. 1. Phantom studies," Med. Phys., vol. 13, no. 3, pp. 334-9, May 1986.

[3] N. H. Clinthorne, "A constrained dual-energy reconstruction method for material-selective transmission tomography," Nucl. Instr. Meth. Phys. Res. A., vol. 353, no. 1, pp. 347-8, Dec. 1994.

[4] P. Sukovic and N. H. Clinthorne, "Penalized weighted leastsquares image reconstruction for dual energy X-ray transmission tomography," IEEE Trans. Med. Imag., vol. 19, no. 11, pp. 1075-81, Nov. 2000.

[5] J. A. Fessler, I. Elbakri, P. Sukovic, and N. H. Clinthorne, "Maximum-likelihood dual-energy tomographic image reconstruction," in Proc. SPIE 4684, Medical Imaging 2002: Image Proc., 2002, vol. 1, pp. 38-49.

[6] J. A. O'Sullivan and J. Benac, "Alternating minimization algorithms for transmission tomography," IEEE Trans. Med. Imag., vol. 26, no. 3, pp. 83-297, Mar. 2007.

[7] D. L. Snyder, C. W. Helstrom, A. D. Lanterman, M. Faisal, and R. L. White, "Compensation for readout noise in CCD images," J. Opt. Soc. Am. A, vol. 12, no. 2, pp. 272-83, Feb. 1995.
[8] C. Ruth and P. M. Joseph, "Estimation of a photon energy spectrum for a computed tomography scanner," Med. Phys., vol. 24, no. 5, pp. 695-702, May 1997.

[9] A. P. Colijn and F. J. Beekman, "Accelerated simulation of cone beam X-ray scatter projections," IEEE Trans. Med. Imag., vol. 23, no. 5, pp. 584-90, May 2004.

[10] J. H. Hubbell and S. M. Seltzer, “Tables of X-ray mass attenuation coefficients and mass energy-absorption coefficients," 1995, National Institute of Standards and Technology http://physics.nist.gov/PhysRefData/XrayMassCoef.

[11] J. Hsieh, "Adaptive streak artifact reduction in computed tomography resulting from excessive x-ray photon noise," Med. Phys., vol. 25, no. 11, pp. 2139-47, Nov. 1998.

[12] P. Charbonnier, L. Blanc-Féraud, G. Aubert, and M. Barlaud, "Two deterministic half-quadratic regularization algorithms for computed imaging," in Proc. IEEE Intl. Conf. on Image Processing, 1994, vol. 2, pp. 168-71.

[13] J. A. Fessler and W. L. Rogers, "Spatial resolution properties of penalized-likelihood image reconstruction methods: Spaceinvariant tomographs," IEEE Trans. Im. Proc., vol. 5, no. 9, pp. 1346-58, Sept. 1996.

[14] J. A. Fessler and S. D. Booth, "Conjugate-gradient preconditioning methods for shift-variant PET image reconstruction," IEEE Trans. Im. Proc., vol. 8, no. 5, pp. 688-99, May 1999.

[15] H. Erdoğan and J. A. Fessler, "Ordered subsets algorithms for transmission tomography," Phys. Med. Biol., vol. 44, no. 11, pp. 2835-51, Nov. 1999. 